\title{
The Riccati-Bernoulli Sub-ODE Technique for Solving the Deterministic (Stochastic) Generalized-Zakharov System
}

\author{
Mahmoud A.E. Abdelrahman, M. A. Sohaly \\ Department of Mathematics, Faculty of Science, Mansoura University, 35516 Mansoura, Egypt
}

\section{ABSTRACT}

This article concerns with the construction of the analytical traveling wave solutions for the Generalized -Zakharov System by the Riccati-Bernoulli Sub- ODE technique. Also, we will discuss this technique in random case by using random traveling wave trans- formation in order to find what is the effect of the randomness input for this technique. We presented the Generalized-Zakharov System as an example to show the difference effect between the deterministic and stochastic Riccati-Bernoulli Sub-ODE technique. The first moment of random solution is computed for different statistical probability distributions.

Keywords: Riccati-Bernoulli Sub-ODE Method; Generalized-Zakharov System; (Stochastic) Traveling Wave Solutions; (Stochastic) Solitary Wave Solutions; Random Variable

\section{Introduction}

Over the years, mathematicians and physicists gave their attention to find exact solutions of nonlinear partial differential equations (NPDEs). In fact these equations are resulted from non-linear phenomena, which arise in many areas of science, for example fluid mechanics, biology, optical fibers, plasma physics, chemical kinematics see. ${ }^{[1-11]}$ Thus, many new methods have been proposed to investigate these equations, such as the tanh-sech method ${ }^{[12-14]}$, Jacobi elliptic function $\operatorname{method}^{[15-17]}$, exp-function method $\left.{ }^{[18,19}\right]$, sine-cosine $\operatorname{method}^{[20-22]}$, homogeneous balance $\operatorname{method}^{[23,24]}$, F-expansion method ${ }^{[25-27]}$, extended tanh-method ${ }^{[28-30]}$, ( G )-expansion method ${ }^{[31,32]}$ and so on.

In this paper we consider the generalized Zakharov equations (GZEs) ${ }^{[33]}$ in the form

$$
\begin{array}{r}
i \chi t+\chi x x-2 \lambda|\chi| 2 \chi+2 \chi v=0 \\
v t t-v x x+(|\chi| 2) x x=0
\end{array}
$$

where $\chi(x, t)$ is the complex envelope of the high-frequency wave and $v(x, t)$ is the real lowfrequency eld. The cubic term in the above equation of 3.19 describes the nonlinear self- interaction in the high frequency subsystem, such a term corresponds to a self-focusing effect in plasma physics. The coefficient $\lambda$ is a real constant that can be a positive or negative number. When $\lambda=0$, (3.19) reduced to the classical Zakharov equation of plasma physics. Obviously, this system is a strongly nonlinear and it is quiet difficult to obtain its solitary wave solutions. In our paper, we used the Riccati-Bernoulli sub-ODE $\operatorname{method}^{[10,11,34]}$ to construct exact solutions, solitary wave solutions of NPDEs. By using a traveling wave transformation and the Riccati-Bernoulli equation, these equations can be transferred to a set of algebraic equations. We can get the exact solutions of NPDEs, by solving these equations. The generalized Zakharov equations are chosen to illustrate the validity of this method.

Simulation by the deterministic model can be considered one of the interested instances of simulation by the stochastic model. In other words, when there are no random inputs in the deterministic model, simulation can well be done just one. We can deal with stochastic models as the problem we want to solve, $\operatorname{se}^{[10,35,36]}$ or by using randomtechnique as $\mathrm{in}^{[37]}$. In this paper we will use the random Riccati-Bernoulli Sub-for solving generalized-Zakharov System.Surely, when we deal with stochastic case the method will be complicated than deterministic case. In this work, we are concerning with the random solutions by using different statistical distributions and find some statistical moments.

Copyright (C) 2018 Mahmoud A.E. Abdelrahman et al.

doi: 10.24294/ijmss.v1i3.810

EnPress Publisher LLC.This work is licensed under the Creative Commons Attribution-NonCommercial 4.0 International License (CC BY-NC 4.0). http://creativecommons.org/licenses/ by/4.0/ 
The rest of the paper is arranged as follows: the Riccati-Bernoulli sub-ODE method is described in Section 2. In Section 3, in order to illustrate the method, generalized-Zakharov System is investigated and abundant exact solutions are obtained which include new solitary wave solution. Our results in random case will also be shown with case study in this section. Finally, in Section 4 we give the conclusions.

\section{The Riccati-Bernoulli sub-ODE method}

Consider the following nonlinear evolution equation

$$
\mathrm{P}(\varphi, \varphi \mathrm{t}, \varphi \mathrm{x}, \varphi \mathrm{tt}, \varphi \mathrm{xx}, \ldots .)=0,
$$

where $\mathrm{P}$ is a polynomial in $\varphi(\mathrm{x}, \mathrm{t})$ and its partial derivatives with even highest order derivatives and nonlinear terms. The main steps of this method ${ }^{[38]}$ given as follows:

Step 1. We use the wave transformation

$$
\varphi(\mathrm{x}, \mathrm{t})=\varphi(\xi), \xi=\mathrm{k}(\mathrm{x}+\mathrm{vt}),
$$

where the localized wave solution $\varphi(\xi)$ travels with speed $\mathrm{v}$ and $\mathrm{k}$ is a positive constant. Using equation (2.2), one can transform equation (2.1) into the following ODE:

$$
\mathrm{H}(\varphi, \varphi \mathrm{I}, \varphi \mathrm{II}, \varphi \mathrm{III}, \ldots . .)=0,
$$

where $\mathrm{H}$ is a polynomial in $\varphi(\xi)$ and its total derivatives, while

$$
\phi^{\prime}(\xi)=\frac{d \phi}{d \xi}, \phi^{\prime \prime}(\xi)=\frac{d^{2} \phi}{d \xi^{2}}
$$

and so on.

Step 2. We assume that equation (2.3) has the formal solution in the following form:

$$
\varphi \mathrm{I}=\mathrm{a} \varphi 2-\mathrm{n}+\mathrm{b} \varphi+\mathrm{c} \varphi \mathrm{n}
$$

where $\mathrm{a}, \mathrm{b}, \mathrm{c}$ and $\mathrm{n}$ are constants to be determined in sequel . From equation (2.4), we get

$\varphi \mathrm{II}=\mathrm{ab}(3-\mathrm{n}) \varphi 2-\mathrm{n}+\mathrm{a} 2(2-\mathrm{n}) \varphi 3-2 \mathrm{n}+\mathrm{nc} 2 \varphi 2 \mathrm{n}-1$

$+\mathrm{bc}(\mathrm{n}+1) \varphi \mathrm{n}+(2 \mathrm{ac}+\mathrm{b} 2) \varphi$,

$\varphi \mathrm{III}=(\mathrm{ab}(3-\mathrm{n})(2-\mathrm{n}) \varphi 1-\mathrm{n}+\mathrm{a} 2(2-\mathrm{n})(3-2 \mathrm{n}) \varphi$ $2-2 \mathrm{n}+\mathrm{n}(2 \mathrm{n}-1) \mathrm{c} 2 \varphi 2 \mathrm{n}-2+\mathrm{bcn}(\mathrm{n}+1) \varphi \mathrm{n}-1+(2 \mathrm{ac}+$ b2)) $\varphi \mathrm{I}$,

Remark 2.1. When $\mathrm{ac}=0$ and $\mathrm{n}=0$, equation (2.4) is a Riccati equation. When $\mathrm{a}=0, \mathrm{c}=0$, and $\mathrm{n}=0$, equation (2.4) is a Bernoulli equation. Equation (2.4) is called the Riccati-Bernoulli equation.

\subsection{Classification of the solutions}

Here we give the cases of solutions for the Riccati-Bernoulli equation (2.4).

Case 1: When $n=1$, the solution of equation (2.4) is

$$
\varphi(\xi)=\mu \mathrm{e}^{(\mathrm{a}+\mathrm{b}+\mathrm{c}) \xi}
$$

Case 2: When $\mathrm{n}=1, \mathrm{~b}=0$ and $\mathrm{c}=0$, the solution of equation (2.4) is

$$
\varnothing(\xi)=(a(n-1)(\xi+\mu))^{\frac{1}{n-1}}
$$

Case 3: When $n=1, b=0$ and $c=0$, the solution of equation (2.4) is

$$
\emptyset(\xi)=\left(\frac{-a}{h}+\mu e^{b(n-1) \xi}\right)^{\frac{r}{n-1}}
$$

Case 4: When $n=1, a=0$ and $b^{2}-4 a c<0$, the solution of equation (2.4) is

$$
\varnothing(\xi)=\left(\frac{-a}{b}+\frac{\sqrt{4 a c-b^{2}}}{2 a} \tan \left(\frac{(1-n) \sqrt{4 a c-b^{2}}}{2}(\xi+\mu)\right)\right)^{\frac{1}{1-n}}
$$

and

$$
\begin{gathered}
\varnothing(\xi)=\left(\frac{-b}{2 a}-\frac{\sqrt{4 a b-b^{2}}}{2 a} \cot \left(\frac{(1-n) \sqrt{4 a c-b^{2}}}{2}\right)(\xi\right. \\
+\mu))^{\frac{1}{1-n}}
\end{gathered}
$$

Case 5: When $\mathrm{n} /=1, \mathrm{a} \quad /=0$ and $\mathrm{b} 2-4 \mathrm{ac}>0$, the solution of equation (2.4) is

$$
\begin{gathered}
\emptyset(\xi)=\left(\frac{-b}{2 a}-\frac{\sqrt{b^{2}-4 a c}}{2 a} \operatorname{coth}\left(\frac{(1-n) \sqrt{b^{2}-4 a c}}{2}\right)(\xi\right. \\
+\mu))^{\frac{1}{1-n}}
\end{gathered}
$$

and

$\emptyset(\xi)$

$$
\begin{aligned}
& =\left(\frac{-b}{2 a}-\frac{\sqrt{b^{2}}-4 a c}{2 a} \tanh \left(\frac{(1-n) \sqrt{b^{2}-4 a c}}{2}\right)(\xi\right. \\
& +\mu))^{\frac{1}{1-n}}
\end{aligned}
$$

Case 6: When $\mathrm{n} /=1$, a $/=0$ and $\mathrm{b} 2-4 \mathrm{ac}=0$, the solution of equation (2.4) is

$$
\varnothing(\xi)=\left(\frac{1}{a(n-1)(\xi+\mu)}-\frac{b}{2 a}\right)^{\frac{1}{1-n}}
$$

where $\mu$ is an arbitrary constant.

Step 3. superseding the derivatives of $\mathrm{u}$ into equation (2.3) gives an algebraic equation of $u$. Noticing the symmetry of the right-hand item of equation (2.4) and setting the highest power exponents of $\mathrm{u}$ to 
equivalence in equation (2.3), $\mathrm{n}$ can be determined. Comparing the coefficients of ui yields a set of algebraic equations for $\mathrm{a}, \mathrm{b}, \mathrm{c}$, and $\mathrm{v}$. Solving these algebraic equations and substituting $\mathrm{n}, \mathrm{a}, \mathrm{b}, \mathrm{c}, \mathrm{v}$, and $\xi=\mathrm{k}(\mathrm{x}+\mathrm{vt})$ into equations (2.7)-(2.14)), we have traveling wave solutions of equation (2.1).

\section{Applications}

\subsection{The Generalized-Zakharov System in Deterministic Case}

Here we apply Riccat-Bernoulli Sub-ODE method to solve the generalized-Zakharov system (3.19).

Using the traveling wave transformation

$$
\begin{gathered}
\chi(\mathrm{x}, \mathrm{t})=\mathrm{e}^{\mathrm{i} \omega} \varphi(\xi), \\
\quad v(\mathrm{x}, \mathrm{t})=v(\xi), \quad \omega=\alpha \mathrm{x}+\beta \mathrm{t}, \quad \xi= \\
\mathrm{k}(\mathrm{x}-2 \alpha \mathrm{t}),
\end{gathered}
$$

where $\varphi(\xi)$ and $v(\xi)$ are real functions and $\alpha, \beta$ and $\mathrm{k}$ are real constants.

Substituting (3.20) into equations (3.19), we have

$$
\begin{aligned}
\mathrm{k}^{2} \varphi^{\prime}+2 \varphi v-\left(\alpha^{2}+\beta\right) \varphi-2 \lambda \varphi^{3} & =0 . \\
\mathrm{k}^{2}\left(4 \alpha^{2}-1\right) v^{\prime}+\mathrm{k}^{2}\left(\varphi^{2}\right) " & =0 .
\end{aligned}
$$

Integrating equation (3.2) twice with respect to $\xi$ once, and neglecting the constant of inte- gration, we have

$$
v(\xi)=\varphi^{2} /\left(1-4 \alpha^{2}\right) \text { if } \quad \varphi^{2}=1 / 4
$$

Substituting equation (3.4) into equation (3.3), we obtain

$$
\mathrm{k}^{2} \varphi^{\prime \prime}-\left(\alpha^{2}+\beta\right) \varphi+2\left(1 /\left(1-4 \alpha^{2}\right)-\lambda\right) \varphi 3=0 .
$$

Substituting equations (2.5) into equation (3.5), we obtain

$\mathrm{k}^{2}\left(\mathrm{ab}(3-\mathrm{m}) \varphi^{2-\mathrm{m}}+\mathrm{a}^{2}(2-\mathrm{m}) \varphi^{3-2 \mathrm{~m}}+\mathrm{mc}^{2} \varphi^{2 \mathrm{~m}-1}+\mathrm{bc}(\mathrm{m}+1) \varphi^{\mathrm{m}}\right.$ $\left.+\left(2 \mathrm{ac}+\mathrm{b}^{2}\right) \varphi\right)-\left(\alpha^{2}+\beta\right) \varphi+2\left(1 /\left(1-4 \alpha^{2}\right)-\lambda\right) \varphi 3=0$.

Setting $\mathrm{m}=0$, equation (3.6) is reduced to

$\mathrm{k}^{2}\left(3 \mathrm{ab} \varphi+2 \mathrm{a}^{2} \varphi^{3}+\mathrm{bc}+\left(2 \mathrm{ac}+\mathrm{b}^{2}\right) \varphi\right)-\left(\alpha^{2}+\beta\right) \varphi+2(1 /(1-$ $\left.\left.4 \alpha^{2}\right)-\lambda\right) \varphi 3=0$.

Setting each coefficient of $\varphi \mathrm{i}(\mathrm{i}=0,1,2,3)$ to zero, we get

$$
\begin{gathered}
\mathrm{k}^{2} \mathrm{bc}=0, \\
\mathrm{k} 2(2 \mathrm{ac}+\mathrm{b} 2)-(\alpha 2+\beta)=0, \\
3 \mathrm{k}^{2} \mathrm{ab}=0, \\
\mathrm{k}^{2} \mathrm{a}^{2}+\left(1 /\left(1-4 \alpha^{2}\right)-\lambda\right)=0 .
\end{gathered}
$$

Solving equations (3.8)-(3.11), we get

$$
\begin{gathered}
\mathrm{b}=0, \\
\mathrm{ac}=\left(\alpha^{2}+\beta\right) / 2 \mathrm{k}^{2}, \\
\mathrm{a}= \pm\left(\left(\lambda-1+4 \alpha^{2}\right) / \mathrm{k}^{2}\left(1-4 \alpha^{2}\right)\right)^{1 / 2},
\end{gathered}
$$

Case I. When $\left(\alpha^{2}+\beta\right)>0$, substituting equations (3.12)-(3.14) and (3.20) into equations (2.10) and (2.11), we get exact traveling wave solutions of equation (3.19), $\emptyset_{1,2}(x, t)= \pm$

$$
\begin{aligned}
& \sqrt{\frac{\left(\alpha^{2}+\beta\right)\left(1-4 \alpha^{2}\right)}{2\left(\lambda-1+4 \alpha^{2}\right)} \tan \left(\sqrt{\frac{\alpha^{2}+\beta}{2 k^{2}}}(k(x-2 \alpha t)+\mu)\right)} \\
& \emptyset_{3,4}(x, t)= \pm \\
& \sqrt{\frac{\left(\alpha^{2}+\beta\right)\left(1-4 \alpha^{2}\right)}{2\left(\lambda-1+4 \alpha^{2}\right)} \operatorname{and}\left(\sqrt{\frac{\alpha^{2}+\beta}{2 k^{2}}}(k(x-2 \alpha t)+\mu)\right)}
\end{aligned}
$$

where $\alpha, \beta, \mathrm{k}$, and $\mu$ are arbitrary constants. The solution $\varphi 1$ is depicted in Figure 1.

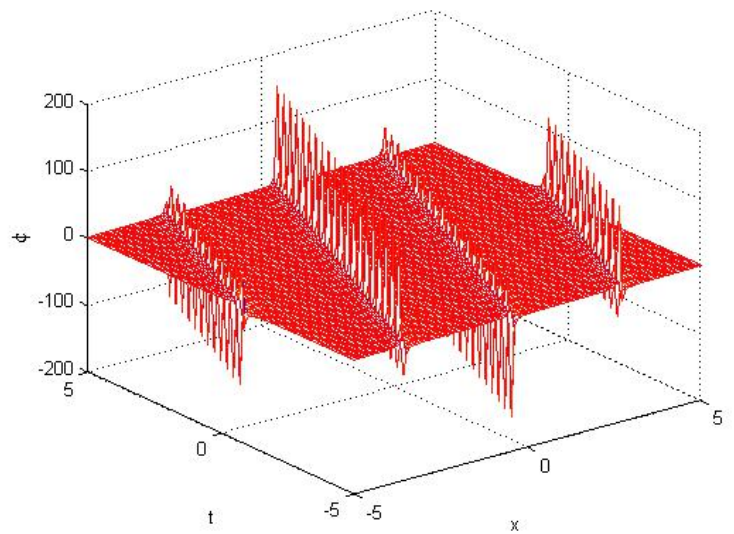

Figure 1; Graph of solution $\varphi=\varphi 1(\mathrm{x}, \mathrm{t})$ of the generalized-Zakharov system for $\alpha=0.2, \beta=2$.

$$
\lambda=2.5, \mathrm{k}=2, \mu=0 \text { and }-5 \leqslant \mathrm{t}, \mathrm{x} \leqslant 5 .
$$

Case II. When $(\alpha 2+\beta)<0$, substituting equations (3.12)-(3.14) and (3.20) into equations (2.12) and (2.13), we get exact traveling wave solutions of equation (3.19),

$$
\begin{aligned}
& \emptyset_{5,6}(x, t)= \pm \\
& \frac{\left(\alpha^{2}+\beta\right)\left(4 \alpha^{2}-1\right)}{2\left(\lambda-1+4 \alpha^{2}\right)} \tanh \left(\sqrt{-\frac{\alpha^{2}+\beta}{2 k^{2}}}(k(x-2 \alpha t)+\mu)\right)
\end{aligned}
$$

and

$$
\frac{\emptyset_{7.8}(x, t)= \pm}{\sqrt{\frac{\left(\alpha^{2}+\beta\right)\left(4 \alpha^{2}-1\right)}{2\left(\lambda-1+4 \alpha^{2}\right)} \operatorname{coth}\left(\sqrt{-\frac{\alpha^{2}+\beta}{2 k^{2}}}(k(x-2 \alpha t)+\mu)\right)}}
$$

where $\alpha, \beta$ and $\zeta$, and $\mu$ are arbitrary constants. The 
solution $\varphi 5$ is depicted in Figure 2.

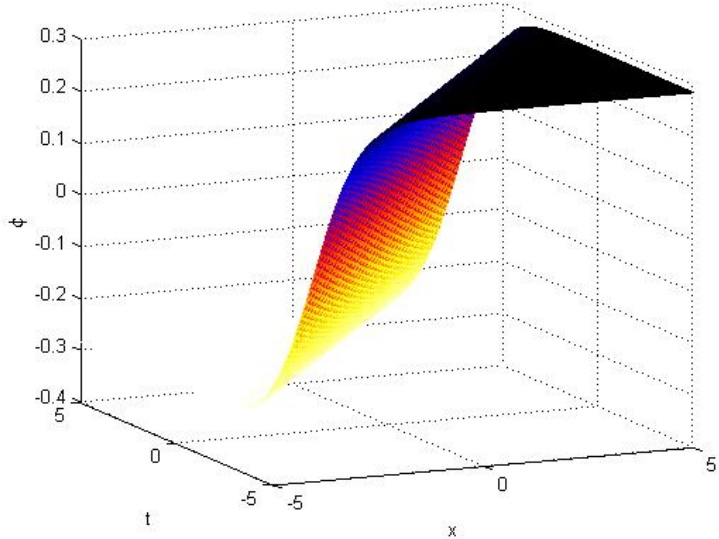

Figure 2; Graph of solution $\varphi=\varphi 5(x, t)$ of the generalized-Zakharov system for $\alpha=0.4, \beta=-2, \lambda=4.5, \mathrm{k}=2, \mu$ $=0$ and $-5 \leqslant \mathrm{t}, \mathrm{x} \leqslant 5$.

\subsection{The Generalized-Zakharov System in Stochastic Case}

A deterministic model assumes that its outcome is certain when the input to the model is fixed. No matter how many times one recalculates, one obtains exactly the result itself. It is arguable that the random model is more informative than a deterministic model since the former accounts for the uncertainty due to varying behavioural characteristics.

In our life, a deterministic model is one where the model parameters are known or assumed.Deterministic models describe behaviour on the main of some physical law.

Deterministic models are often developed by statistical techniques such as linear regression or non-linear curve fitting procedures that essentially model the average system behaviour of an equilibrium or steady state relationship. In a live transportation system, a totally deterministic model is unlikely to involve different dynamic random effects (or uncertainties). The uncertainty is usually understood as factors related to imperfect knowledge of the system under concern, especially those being random in nature. It is closely related to heterogeneity, that denotes the state since entities within a given system are of non-uniform character. For example, when the heterogeneity is not faithfully recognized, the uncertainty increases. Conversely, a decrease in uncertainty denotes that the system is better understood and thus the heterogeneity is better recognized.
In this part we want to develop the The Generalized-Zakharov System from deterministic case to random case by adding some disturbance in the system itself as follow:

$$
\begin{gathered}
{ }^{\mathrm{i}} \chi \mathrm{t}+\chi_{\mathrm{xx}}-2 \lambda|\chi| 2 \chi+2 \chi v=0, \\
v_{\mathrm{tt}}-v_{\mathrm{xx}}+\left(|\chi|^{2}\right)_{\mathrm{xx}}=0,
\end{gathered}
$$

where $\lambda$ is a random variable.

Additionally, we can develop the Riccat-Bernoulli Sub-ODE method from deterministic tech- nique to random technique as follow:

when we using the random traveling wave transformation as follow:

$\chi(x, t)=\operatorname{ei\omega \varphi }(\xi), v(x, t)=v(\xi), \omega=\alpha x+\beta t, \xi=\gamma(x-2 \alpha t)$,

where $\varphi(\xi)$ and $v(\xi)$ are functions of random variables $\alpha$, $\beta$ and $\gamma$.

But, only in this paper we will deal with random traveling wave transformation when we take $\alpha, \beta$ and $\gamma$ are random variables.

So, we can conclude our solution to the Generalized-Zakharov System by using random RiccatBernoulli Sub-ODE technique only, in Case I. When $\left(\alpha^{2}\right.$ $+\beta)>0$ as follow:

$$
\begin{aligned}
& \Psi_{1}(x, t)= \\
& \sqrt{\frac{\left(\alpha^{2}+\beta\right)\left(1-4 \alpha^{2}\right)}{2\left(\lambda-1+4 \alpha^{2}\right)} \tan \left(\sqrt{\frac{\alpha^{2}+\beta}{2 \Upsilon^{2}}}(\Upsilon(x-2 \alpha t)+\mu)\right)} \\
& \text { and }
\end{aligned}
$$

$$
\begin{aligned}
& \Psi_{3}(x, t)= \\
& \sqrt{\frac{\left(\alpha^{2}+\beta\right)\left(1-4 \alpha^{2}\right)}{2\left(\lambda-1+4 \alpha^{2}\right)} \cot \left(\sqrt{\frac{\alpha^{2}+\beta}{2 \Upsilon^{2}}}(\Upsilon(x-2 \alpha t)+\mu)\right)}
\end{aligned}
$$

where $\alpha, \beta, \gamma$ are random variables and $\mu$ is arbitrary constant. The random solutions $\psi 1, \psi 3$ are depicted in Figures 3 and 4 by different statistical distributions.

Proposition 3.1. ${ }^{[35,37]}$ To obtain convergent random Riccat-Bernoulli Sub-ODE solution we must take these conditions in our consideration:

1. All derivatives in (2.1) are bounded.

2. $\alpha, \beta, \gamma$ are positive and bounded random variables.

3. $\mathrm{E}[\alpha]^{2}<\infty, \mathrm{E}[\beta]^{2}<\infty$ and $\mathrm{E}[\gamma]^{2}<\infty$. 


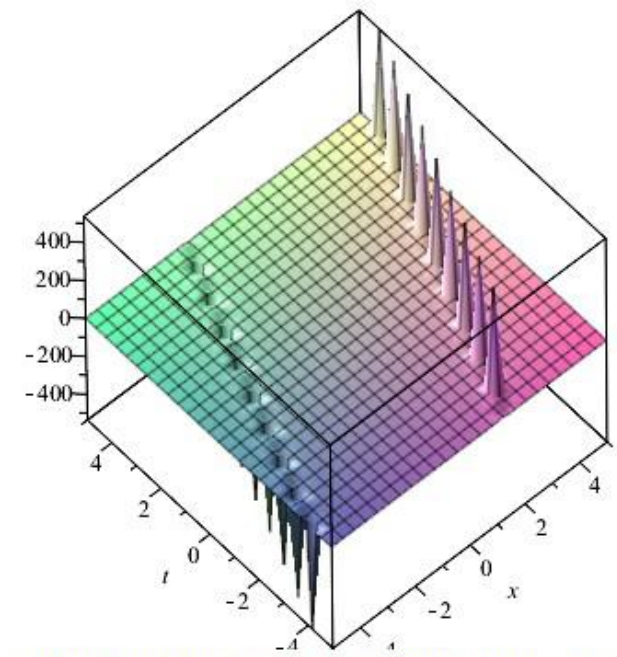

Graph of solution $\psi_{1}$ with $\alpha, \beta \sim \mathrm{Be}(0.5,0.5)$, and $\gamma \sim \mathrm{Be}(0.001$, $0.005), \lambda=2.5$ and $\mu=0$

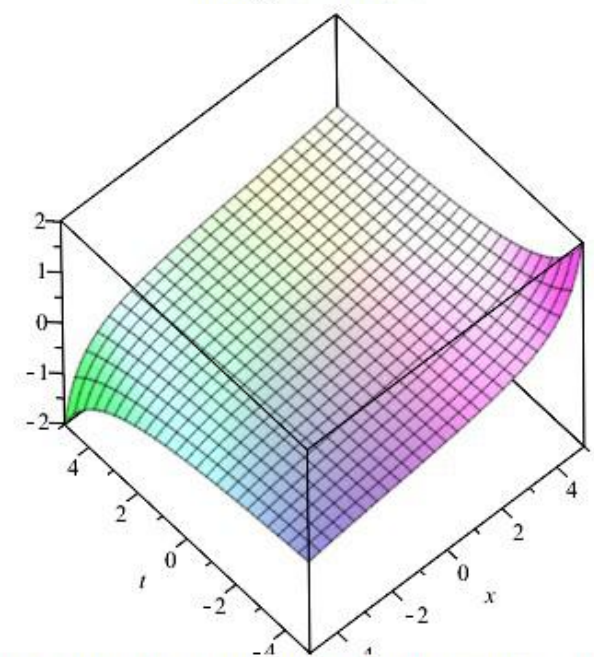

Graph of solution $\psi_{1}$ with $\alpha, \beta \sim \mathrm{G}$ amma $(0.5,0.5)$, and $\gamma \sim \mathrm{G}$ amma $(0.001,0.005), \lambda=2.5$ and $\mu=0$

Figure 3; Graph of solution $\mathrm{E}[\psi]=\mathrm{E}[\psi 1(\mathrm{x}, \mathrm{t})]$ of the system (3.19) .

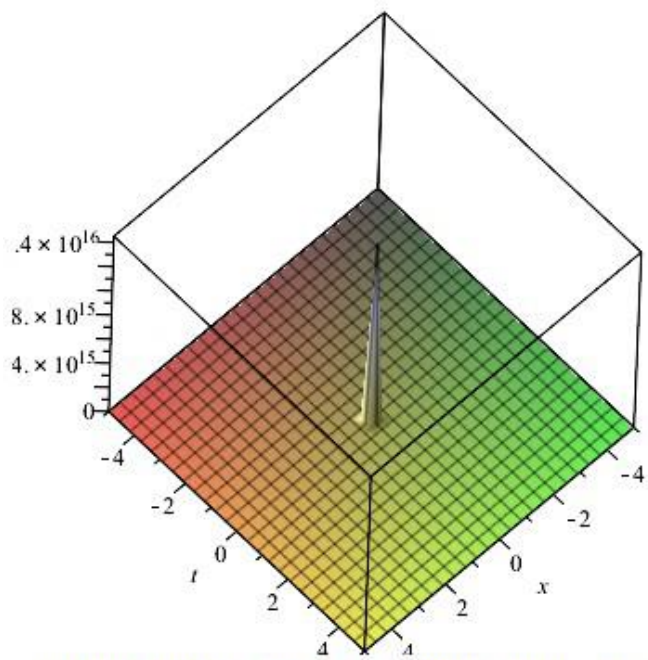

Graph of solution $\psi_{3}$ with $\alpha, \beta \sim \mathrm{Be}(0.5,0.5)$, and $\gamma \sim \mathrm{Be}(0.001$, $0.005), \lambda=2.5$ and $\mu=0$

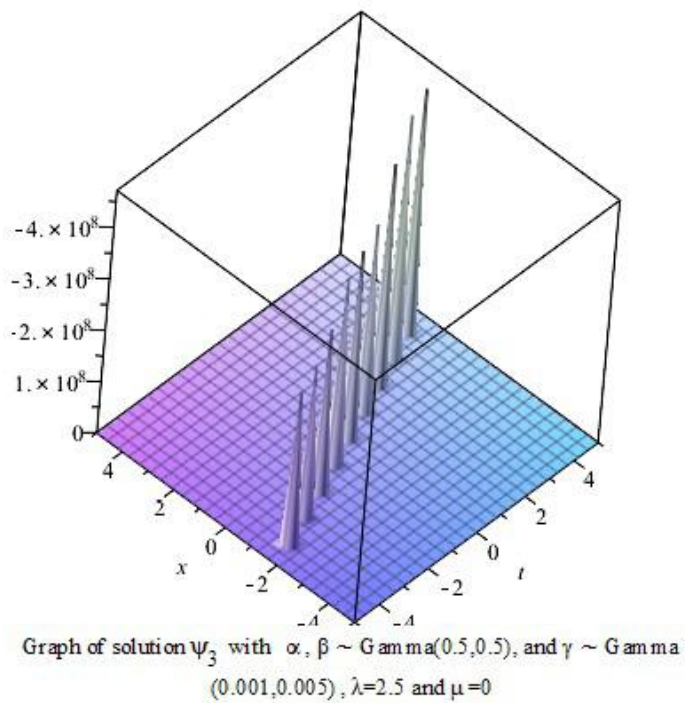

Figure 4; Graph of solution $\mathrm{E}[\psi]=\mathrm{E}[\psi 3(\mathrm{x}, \mathrm{t})]$ of the system (3.19).

\section{Conclusions}

We consider the solution of the Generalized-Zakharov System by using the Riccati-Bernoulli Sub- ODE technique. This method is based on the wave transformation that may be deterministic or involve randomness input. An estimated (random) solution for solving Generalized-Zakharov System is obtained in some cases. The Riccati-Bernoulli Sub-ODE method can give a new infinite sequence of deterministic solutions, which is consideredas an interesting feature of this method. The prominence and merit of our method is that it is not limited to a specific form or problem. In fact, many (random) differential equations can be solved with it. It, also, provides some results with high accuracy and also, with the least amount of error.

\section{References}

1. Abdelrahman MAE, Kunik M. The interaction of waves for the ultra-relativistic Euler equations, J. Math. Anal. Appl. 2014; 409: 1140-1158.

2. Abdelrahman MAE, Kunik M. The Ultra-Relativistic Euler Equations, Math. Meth. Appl. Sci.38 2015;1247- -1264.

3. Abdelrahman MAE. Global Solutions for the Ultra-Relativistic Euler Equations, Nonlinear Analysis 2017; 155: $140-162$.

4. Abdelrahman MAE. On the Shallow Water Equations, Z. Naturforsch., 72(9)a 2017; 873-879.

5. Abdelrahman MAE. Numerical investigation of the wave-front tracking algorithm for the full ultra-relativistic Euler equations, International Journal of Nonlinear Sciences and Nu- merical 
Simulation DOI:

https://doi.org/10.1515/ijnsns-2017-0121.

6. Razborova P, Ahmed B, Biswas A. Solitons, shock waves and conservation laws of

Rosenau-KdV-RLW equation with power law nonlinearity. Appl. Math. Inf. Sci. 2014; 8(2): 485-491.

7. Biswas AQ, Mirzazadeh M. Dark optical solitons with power law nonlinearity using (G'/G)-expansion.Optik 2014; 125: 4603-4608.

8. Younis M, Ali S, Mahmood SA. Solitons for compound $\mathrm{KdV}$ Burgers equation with variable coefcients and power law nonlinearity. Nonlinear Dyn. 2015; 81: 1191-1196.

9. Bhrawy AH. An efficient Jacobi pseudo spectral approximation for nonlinear complex gen- eralized Zakharov system. Appl. Math. Comput.2014; 247: 30-46.

10. Abdelrahman MAE, Sohaly MA. Solitary Waves for the Modified Korteweg-De Vries Equation in Deterministic Case and Random Case. J Phys Math. 2017; 8(1), [DOI:10.4172/2090-0902.1000214].

11. Abdelrahman MAE, Sohaly MA. Solitary waves for the nonlinear Schro" dinger problem with theprobability distribution function in stochastic input case. Eur. Phys. J. Plus 2017.

12. Malfliet W. Solitary wave solutions of nonlinear wave equation, Am. J. Phys.1992; 60: 650-654.

13. Malfliet W. Hereman W, The tanh method: Exact solutions of nonlinear evolution and wave equations, Phys.Scr. 1996; 54: 563-568.

14. Wazwaz AM. The tanh method for travelling wave solutions of nonlinear equations, Appl.Math. Comput., 2004; 154: 714-723.

15. Dai CQ, Zhang JF. Jacobian elliptic function method for nonlinear differential difference equations, Chaos Solutions Fractals, 2006; 27 : 1042-1049.

16. Fan E, Zhang J. Applications of the Jacobi elliptic function method to special-type nonlinear equations, Phys. Lett. A 2002; 305: 383-392.

17. Liu S, Fu Z, et al. Jacobi elliptic function expansion method and periodic wave solutions of nonlinear wave equations, Phys. Lett. A 2001; 289: 69-74.

18. He JH, Wu XH. Exp-function method for nonlinear wave equations, Chaos Solitons Fractals 2006; 30: 700-708.

19. Aminikhad H, Moosaei H, Hajipour M. Exact solutions for nonlinear partial differential equations viaExp-function method, Numer. Methods Partial Differ. Equations 2009; 26: 1427-1433.

20. Wazwaz AM. Exact solutions to the double sinh-Gordon equation by the tanh method and a variable separated ODE. Method, Comput. Math. Appl., 2005; 50: 1685-1696.

21. Wazwaz AM. A sine-cosine method for handling nonlinear wave equations, Math. Comput.Modelling 2004; 40: 499-508.

22. Yan C. A simple transformation for nonlinear waves, Phys. Lett. A 1996; 224: 77-84.

23. Fan E, Zhang H. A note on the homogeneous balance method, Phys. Lett. A 1998; 246: 403-406.

24. Wang ML. Exct solutions for a compound KdV-Burgers equation, Phys. Lett. A 1996; 213 : 279-287.

25. Abdou MA. The extended F-expansion method and its application for a class of nonlinear evolution equations, Chaos Solitons Fractals 2007; 31: 95-104.

26. Ren YJ, Zhang HQ. A generalized F-expansion method to find abundant families of Jacobi elliptic function solutions of the (2+1)-dimensional Nizhnik-Novikov-Veselov equation, Chaos Solitons Fractals 2006; 27: 959-979.

27. Zhang JL, Wang ML, Wang YM, et al. The improved F-expansion method and its applications, Phys.Lett.A 2006; 350: 103-109.

28. EL-Wakil SA, Abdou MA. New exact travelling wave solutions using modified extented tanh-function method, Chaos Solitons Fractals 2007; 31: 840-852.

29. Fan E. Extended tanh-function method and its applications to nonlinear equations, Phys. Lett. A 2000; 277: 212-218.

30. Wazwaz AM. The extended tanh method for abundant solitary wave solutions of nonlinear wave equations, Appl. Math. Comput. 2007; 187: 1131-1142.

31. Wang ML, Zhang JL, XZ LI. $\left(\frac{G^{\prime}}{G}\right)$-expansion method and travelling wave solutions of nonlinear evolutions equations in mathematical physics, Phys. Lett. A 2008; 372: 417-423.

32. Zhang S, Tong JL, Wang W. Ageneralized $\left(\frac{G^{\prime}}{G}\right)$-expansion method for the mKdv equation with variable coefficients, Phys. Lett. A 2008; 372: 2254-2257.

33. El-Wakil SA, Abdou MA, Elhanbaly A. Phys. Lett. A 2006; 353: 40.

34. Abdelrahman MAE. A note on Riccati-Bernoulli Sub-ODE method combined with complex transform method applied to fractional differential equations, Nonlinear Engineering Modeling and Application (to appear).

35. Sohaly M. Mean Square Heuns Method Convergent for Solving Random Differential Initial Value Problems of First Order. American Journal of Computational Mathematics 2014; 4: 474-481. doi: 10.4236/ajcm.2014.45040.

36. Yassen M, Sohaly M, Elbaz I. Random Crank-Nicolson Scheme for Random Heat Equation in Mean Square Sense. American Journal of Computational Mathematics 2016; 6: 66-73. doi: 10.4236/ajcm.2016.62008

37. Sohaly MA, Yassen MT,Elbaz IM. The Variational Methods for Solving Random Models, International Journal of Innovative Research in Computer 
Science \& Technology (IJIRCST) 2017; 5(2):

2347-5552.

38. Yang XF, Deng ZC, Wei Y. A Riccati-Bernoulli

sub-ODE method for nonlinear partial differential

equations and its application, Adv. Diff. Equa. 2015;

1: $117-133$. 